\title{
AVALIAÇÃO DAS REAÇÕES DO MERCADO DE CAPITAIS NO BRASIL E NA AUSTRÁLIA APÓS O ACIDENTE AMBIENTAL DA MINERADORA SAMARCO
}

\author{
CAPITAL MARKET REACTIONS IN BRAZIL AND AUSTRALIA AFTER THE \\ ENVIRONMENTAL ACCIDENT OF SAMARCO MINING COMPANY
}

\section{FRANCISCO SÁVIO MAURICIO ARAUJO}

Universidade Federal do Ceará. Endereço: Av. Vital Brasil, 2109, Granja Portugal | 60541-495 / Fortaleza/CE | Brasil. (1) http://orcid.org/0000-0002-5965-9948

E-mail: franciscosavio@outlook.com

\section{RÔMULO ALVES SOARES}

Universidade Federal do Ceará. Endereço: Rua Tenente Roma, 157 / Aerolândia | 60851030 | Fortaleza/CE | Brasil.

(1) http://orcid.org/0000-0003-2412-3796

E-mail: romuloalves61@gmail.com

\author{
MÔNICA CAVALCANTI SÁ DE ABREU \\ 2470 - Benfica | 60.020-180 | Fortaleza/CE / Brasil. \\ (1) http://orcid.org/0000-0001-9408-5604 \\ E-mail: mabreu@ufc.br
}

Universidade Federal do Ceará. Endereço: Av. Da Universidade,

\section{RESUMO}

O artigo analisa a reação do mercado de capitais após o acidente ambiental da mineradora Samarco. A Samarco se organiza como joint venture societária dividida igualmente entre a empresa brasileira Vale e a empresa anglo-australiana BHP Billiton. Foi adotada a técnica de estudo de eventos para calcular o risco sistemático (beta) e avaliar as variações no retorno das ações de empresas do setor de mineração dos mercados de capitais brasileiro e australiano. Os dados utilizados na pesquisa foram obtidos no BM\&FBovespa para as empresas brasileiras e na Australian Securities Exchange (ASX) para as empresas australianas, ao longo do ano de 2015. Os resultados da pesquisa evidenciam que, no mercado brasileiro, apenas a empresa Vale apresentou um declínio significante do coeficiente beta. Em relação às empresas australianas houve declínio no risco sistemático para as empresas BHP Billiton, Rio Tinto e NCM. O rompimento da barragem de Fundão da mineradora Samarco entrou para o rol dos desastres ambiental globais. No entanto, os impactos econômicos, ambientais e sociais foram rapidamente absorvidos pelo mercado de capitais, e não influenciaram o valor das ações de empresas do setor de mineração no Brasil e na Austrália a longo prazo.

Palavras-chave: Teoria Institucional. Teoria dos Stakeholders. Estudo de Evento. Setor de Mineração. Acidentes Ambientais.

\section{ABSTRACT}

This paper analyzes The article analyzes the reaction of the capital market after the environmental accident of the mining company Samarco. Samarco is organized as a joint venture between the Brazilian company Vale and the Anglo-Australian company BHP Billiton. 
The technique of event study was used to calculate the systematic risk (beta) and to evaluate the variations in the return of the shares of companies of the mining sector of the Brazilian and Australian capital markets. Data for the study were obtained from the BM \& FBOVESPA for Brazilian companies and the Australian Securities Exchange (ASX) for Australian businesses, throughout the year 2015. The survey results show that in Brazil, just Vale company showed a decline beta coefficient. In the case of Australian companies there was a decline in systematic risk for companies BHP Billiton, Rio Tinto and NCM. The disruption of the Samarco mining dam Fundão has entered into the role of global environmental disasters. However, the economic, environmental, and social impacts were quickly absorbed by the capital market, and did not affect the value of the shares of mining companies in the sector in Brazil and Australia in the long run.

Keywords: Institutional Theory. Stakeholder Theory. Event Analysis. Mining Sector. Environmental Accidents.

\section{INTRODUCÃO}

No dia 5 de novembro de 2015, a barragem de Fundão da mineradora Samarco S.A., controlada pelas empresas Vale S.A. e BHP Billiton Brasil Ltda, rompeu e causou o vazamento de cerca de 60 milhões de $\mathrm{m}^{3}$ de lama de rejeitos industriais. Esta lama atingiu os rios da bacia hidrográfica do rio Doce, nos estados de Minas Gerais e Espírito Santo, e desaguou no Oceano Atlântico. O acidente ambiental devastou o distrito de Bento Rodrigues, localizado no município de Mariana (MG), e ocasionou a morte de 19 pessoas. Espíndola, Campos, Lamounir e Silva (2016) reforçam que o impacto do desastre não se restringiu às áreas imediatas à jusante do barramento, mas impactou cursos d'água, terras agricultáveis, atividades econômicas e abastecimento de água, afetando a biodiversidade do Parque Estadual do Rio Doce. Mais de 1.469 hectares de terras foram destruídos e cerca de 1.200 pessoas ficaram desabrigadas.

Fonseca e Fonseca (2016) indicam que o acidente ambiental da mineradora Samarco resultou em recordes globais em termos de volume e distância percorrida de lama tóxica. $\mathrm{O}$ Parque Estadual do Rio Doce, principal remanescente da Mata Atlântica no estado de Minas Gerais, se viu diante de uma ameaça não prevista em seu plano de manejo. Os impactos negativos no ambiente aquático se estenderam por $663 \mathrm{~km}$, até penetrarem nas águas costeiras do estado do Espírito Santo. A população da região enfrentou dificuldades relacionadas, principalmente, à falta de água. Grande parte das cidades atingidas dependiam do abastecimento dos rios da bacia do rio Doce, que se apresentaram impróprios para o consumo humano e animal.

Paradoxalmente, a mineradora Samarco foi reconhecida, nos últimos 20 anos, como uma das líderes em responsabilidade socioambiental no Brasil. Além de ser a primeira mineradora do mundo a ter a certificação ISO 14001 para todas as etapas de produção. No entanto, a pressão por aumento da produção, acrescida às incertezas sobre a capacidade de armazenamento dos resíduos e um ineficiente monitoramento operacional, levou ao rompimento da barragem de Fundão. Fontes e Lopes (2017) explicam que esta barragem é fiscalizada pelo Departamento Nacional de Produção Mineral (DNPM) e foi classificada como categoria de "baixo risco" e "alto dano potencial associado".

Magness (2008) alerta que o foco antropocêntrico exclui as empresas de suas obrigações éticas sobre o meio ambiente natural. As empresas se concentram apenas nas questões definidas dentro do escopo da sua "jurisdição gerencial". A expansão desta jurisdição, para além de questões econômicas e legais, exige a inclusão de forças motrizes arbitrárias ou ideológicas, em direção a uma atuação responsável. A perspectiva da legitimidade leva as empresas a monitorar os valores sociais e atuar de forma a se manterem alinhadas com estes valores. Neste sentido, os stakeholders podem atuar (ou não) na direção de manter a "consciência social" das corporações, que por sua vez desenvolvem (ou não) uma cultura organizacional baseada na ética.

Abreu, Varvakis e Figueiredo (2004) enfatizam que os acidentes ambientais conferem dinamismo à estrutura da indústria. As mudanças ocorrem porque as empresas estão 
submetidas a eventos significativos que exigem respostas na conduta, com reflexos na performance e na própria estrutura de mercado. Os acidentes impactam os fluxos de caixa das empresas (Blanco, Maquieira, \& Lozano, 2009; Klassen \& Mclaughlin, 1996; Yamaguchi, 2008) e aumentam os custos ambientais e sociais (Deegan, 2002; Patten \& Trompeter, 2003; Karpoff, Lott, \& Wehrly, 2005). Pargai e Wheeler (1995) informam que as pressões da comunidade e as sanções informais podem também agravar as penalizações diante de um acidente ambiental.

Tomando por base os atributos de "legitimidade", "urgência" e "poder" estabelecidos no modelo de saliência de Mitchell, Agle e Wood (1997), observa-se que nenhum destes atributos é fixo no tempo, e que acidentes ambientais lhe conferem uma dinâmica. $\mathrm{O}$ acidente ambiental da mineradora Samarco traz à tona a "legitimidade" dos procedimentos operacionais envolvendo o armazenamento dos resíduos de mineração. Em condições normais de operação, os moradores do distrito de Bento Rodrigues não possuem nenhum "poder" para forçar a mineradora Samarco e suas controladoras (Vale e BHP) a se preocuparem com o bem-estar da população ou com a preservação do Parque Estadual do Rio Doce, e para mostrar que existe "urgência" em suas demandas.

Morgan, Gomes e Perez-Aleman (2016) sustentam que o acidente da mineradora Samarco pode ser resultado de uma falha institucional. $\mathrm{Na}$ ausência de uma regulação ambiental e de uma fiscalização eficiente, empresas multinacionais operam sem monitoramento ambiental adequado de suas atividades produtivas, o que coloca em risco a segurança e saúde dos empregados, das comunidades vizinhas e do meio ambiente. Em contraposição à teoria dos stakeholders, a teoria da firma sustenta que acionistas e investidores são os únicos stakeholders que realmente importam, e que são afetados pelo desempenho das empresas ao longo do tempo.

Com base no exposto, este trabalho toma por base a seguinte questão de pesquisa: $O$ mercado de capitais, do setor de mineração do Brasil e da Austrália, reagiu negativamente ao acidente ambiental da mineradora Samarco? A pesquisa utiliza metodologia de "estudo de evento" para avaliar as respostas dos investidores do setor de mineração no Brasil e na Austrália. A avaliação do retorno das ações e a mudança no risco sistemático (beta), que ocorreu desde o primeiro dia após o rompimento da barragem de Fundão até o centésimo dia, permite entender as pressões que levam os tomadores de decisão do setor de mineração a incorporarem (ou não) questões éticas e ambientais em seus modelos de negócio.

Este estudo contribui com a literatura de responsabilidade social corporativa ao mostrar evidências da relação entre elevados retornos financeiros no setor de mineração e uma baixa preocupação com os acidentes ambientais (Kumar, Lamb, \& Wokutch, 2002; Klerk \& Villieres, 2012). O rompimento da barragem de Fundão da mineradora Samarco entrou para o rol dos desastres ambiental globais. No entanto, os impactos econômicos, ambientais e sociais parecem não terem sido suficientes para influenciar imediatamente o valor das ações de empresas do setor de mineração no Brasil e na Austrália.

O artigo está estruturado em seções. Em seguida, são apresentados o efeito do ambiente institucional nas decisões corporativas e o impacto dos acidentes ambientais sobre 0 comportamento de investidores. Na seção da metodologia são descritas as modelagens econométricas adotas no estudo de eventos e os resultados sobre o valor das ações de empresas de mineração na Australian Securities Exchange (ASX) e na BM\&FBovespa. Finalmente, as discussões reforçam a importância do mercado de capitais em promover uma resposta eficiente das empresas em direção à prevenção e à mitigação dos impactos dos acidentes ambientais.

\section{EFEITO DO AMBIENTE INSTITUCIONAL NA TOMADA DE DECISÕES CORPORATIVAS SOCIALMENTE RESPONSÁVEIS}

As empresas geram contribuições para o desenvolvimento sustentável por meio de uma atuação socialmente responsável. A responsabilidade social corporativa (RSC) caracteriza-se pela identificação, antecipação e gerenciamento das expectativas dos stakeholders (Clarkson, 1995). A conceituação da RSC baseia-se em entender como o ambiente institucional e as políticas governamentais afetam a empresa e, portanto, refletem as 
relações empresa-sociedade (Siltaoja \& Onkila 2013). Essas relações podem ser orientadas para o mercado ou não. No entanto, as instituições são necessárias, para encorajar as corporações a responderem a preocupações sociais além de seus próprios interesses econômicos (Campbell, 2007).

O quadro institucional de cada país reflete sua história e as peculiaridades de sua configuração sócio-política (Jamali \& Mirshak, 2007). No entanto, com base na tese comum da globalização, o modelo ocidental de RSC vem sendo introduzido em países emergentes. Neste sentido, um nível "cosmético" de RSC "explícita" pode ser materializado por meio de pressões isomórficas (Jamali \& Neville, 2011). Abreu, Cunha e Barlow (2015) demonstram que os países desenvolvidos têm um conjunto claro de limites e parâmetros que orientam suas respostas de responsabilidade social corporativa. Em contraste, nos países emergentes, os limites são mais desfocados, permitindo que as organizações experimentem novas formulações e definições de sustentabilidade.

A dinâmica institucional e as organizações estão inter-relacionadas. No entanto, as organizações não reagem diretamente a todas as pressões ditadas pelo ambiente organizacional, nem agem de modo completamente autônomo, sem a influência de pressão externa (Hoffman, 2001). Uma variedade de condições institucionais influencia as decisões gerenciais para atuarem de forma socialmente responsável. É mais provável que firmas atuem de forma responsável quando monitoradas por uma forte regulamentação do Estado, ONGs e outras organizações independentes ou que possuam uma autorregulação industrial coletiva. Estes stakeholders podem ser engajados no diálogo e criar pressão sobre as firmas (Campbell, 2007).

As decisões gerenciais são, portanto, fortemente influenciadas por três mecanismos institucionais - isomorfismo coercitivo, mimético e normativo - que criam e difundem um conjunto comum de valores, normas e regras para produzir práticas e estruturas similares entre organizações que compartilham de um mesmo campo (DiMaggio \& Powell, 1983). A teoria institucional enfatiza, portanto, o papel das pressões impostas às instituições, as quais influenciam o campo organizacional em que as empresas atuam (Meyer \& Scott, 1992; Milstein, Hart, \& York, 2002).

Dentro do contexto institucional, existem pressões capazes de influenciar as decisões estratégicas dos gestores, como a pressão exercida pelos órgãos governamentais, que se concretizam por meio de legislações, regulações e outros mecanismos coercitivos. Delmas e Toffel (2004) afirmam que a existência de pressão governamental é importante porque a sociedade, de um modo geral, não tem ciência dos impactos ambientais gerados por atividades produtivas. Henriques e Sadorsky (1996) defendem que sem a devida regulação seria pouco provável que houvesse esforços para amenizar tais impactos.

Outras fontes de pressão, apontadas por Delmas e Toffel (2004), são a comunidade e os consumidores. As comunidades também podem exercer pressões coercitivas sobre as empresas por meio de representação política, ativismo ambiental e ONGs. A pressão da comunidade pode levar o governo a criar mecanismos mais incisivos de proteção e regulação. Isso, por sua vez, pode induzir as empresas que lideram o mercado a adotar uma postura mais ambientalmente proativa. A pressão institucional dos consumidores tem origem no ambiente competitivo em que as empresas atuam. A relação fornecedor-consumidor é o mecanismo principal por meio do qual se desenvolvem atitudes gerenciais e padrões com foco na qualidade dos produtos e serviços.

No caso das empresas de mineração, Lin, Li e Bu (2015) recomendam que, para serem consideradas responsáveis, as empresas devem avaliar os impactos positivos e negativos decorrentes de suas atividades produtivas. Para conseguir a "licença social" para operar, as empresas de mineração devem se engajar com a comunidade. Jennings e Zandbergen (1995) evidenciaram a influência do ambiente institucional na adoção de práticas ambientais, em especial após a ocorrência de desastres ambientais. Neste sentido, Alpaslan, Green e Mitroff (2009) confirmam que "situações de crise" podem afetar a dinâmica da gestão dos stakeholders. 


\subsection{Efeito dos acidentes ambientais sobre o comportamento dos investidores}

Magness (2008) utilizou o modelo de saliência proposto por Mitchell et al. (1997) para investigar as dinâmicas das tomadas de decisões de investidores e gestores, com relação a dois acidentes ambientais envolvendo empresas mineradoras. Na visão de Baskerville-Morley (2004), a legitimidade e o poder são atributos que podem variar dependendo de cada situação. No entanto, a urgência é um atributo "cronológico", ou seja, pode mudar a partir de um evento particular, fixado como um ponto no tempo. Especificamente para os gestores, Magness (2008) encontrou uma percepção do atributo "poder", seguida de um aumento nas exigências regulatórias.

Magness (2000) afirma que os riscos operacionais enfrentados por uma empresa são comuns na indústria. Um acidente ambiental pode intensificar a regulação por parte do governo, o que afetará todas as empresas do setor. Uma regulação governamental mais rígida pode impactar o fluxo de caixa das empresas. Magness (2008) observou uma diferença significativa no comportamento dos investidores em casos de um segundo acidente ambiental envolvendo empresas do mesmo setor industrial. Em virtude do baixo conhecimento sobre o funcionamento da empresa e suas operações, os investidores enxergam o primeiro acidente como uma fatalidade. Entretanto, quando um segundo acidente ocorre, o comportamento muda, e os investidores passam a penalizar, de forma geral, as empresas do setor.

Jenkins e Yakovleva (2006) reconhecem que investidores estão cada vez mais interessados em investigar a dimensão social, ambiental e ética de uma empresa antes de investir nela. No caso da indústria de mineração, divulgar informações sociais e ambientais é crucial para obter uma imagem de "empresa responsável" diante dos investidores. A análise dos relatórios anuais representa, portanto, uma forma de entender em que grau acidentes ambientais mudam procedimentos operacionais e a conduta dos gestores.

Elliott, Wang, Lowe e Kleindorfer (2004) mostram que o nível de atenção de empresas do setor químico aos impactos ambientais, inclusive aqueles gerados por acidentes, está associado ao nível socioeconômico da comunidade circunvizinha. Os autores mostram que as maiores empresas químicas estão situadas próximas a comunidades com um maior nível de desigualdade social. Estas comunidades apresentam uma baixa capacidade de se organizarem e acabam sendo expostas a elevados riscos à saúde e outros efeitos ambientais.

Desastres ambientais, tais como um vazamento de petróleo ou o rompimento de uma barragem de contenção, podem influenciar a percepção dos investidores sobre os fluxos de caixa futuros da empresa. Isto levará a um movimento de compra e venda de ações, influenciando seu preço e, consequentemente, seu risco não sistemático (Bansal \& Clelland, 2009). Jennings e Zandbergen (1995) confirmam que o acidente na usina de Three Mile Island criou uma crise de legitimidade entre as geradoras de energia nuclear nos EUA.

O acidente de Fukushima no Japão causou um impacto global no setor de energia nuclear. Visschers e Siegrist (2012) sugerem que os gestores devem focar na comunicação com o público, sendo transparentes quanto aos riscos que envolvem a energia nuclear e demonstrando suas vantagens de modo a influenciar a opinião pública. Goebel, Krekel, Tiefenbach e Ziebarth (2015) evidenciaram o impacto do acidente nuclear sobre o perfil de consumo de energia dos consumidores japoneses. Antes do acidente, havia uma taxa média de $37 \%$ de consumidores japoneses que deixavam de consumir energia nuclear e passavam a utilizar energia de fontes renováveis. No ano do acidente, esta taxa subiu para $74 \%$, e continuou em $64 \%$ no ano seguinte.

Heflin e Wallace (2015) mostram que, após o vazamento de petróleo da British Petroleum, ocorrido em 2010, os investidores passaram a procurar empresas de petróleo e gás com melhores evidenciações ambientais. Acreditava-se que as empresas estariam melhor preparadas para possíveis mudanças na regulação ambiental, e também por apresentarem um menor risco de acidentes. Em consequência do acidente da BP, houve um aumento na divulgação de informações ambientais, em especial informações sobre os planos de respostas a acidentes ambientais.

O nível de concentração do mercado é também um importante elemento na difusão de práticas ambientais. Se um mercado é dominado por poucos atores, há menos incentivos para o engajamento em ações socialmente responsáveis. Patten (1992) avaliou as respostas das 
empresas petroquímicas americanas após o derramamento de petróleo causado pelo navio Exxon Valdez, no Alaska. A evidenciação ambiental das empresas do setor petroquímico aumentou significativamente após o acidente. A diversidade de empresas de grande porte, no setor de petróleo e gás, influenciou o nível de evidenciação. Na ocorrência de situações extremas, outros stakeholders ganham importância, isto é, maximizar o valor dos shareholders deixa de ser o único objetivo dos CEOs. Este efeito é fortemente observado em ambientes econômicos mais integrados.

Garcia, Ribeiro, Oliveira Roque, Ochoa-Quintero e Laurance (2017) calculam que os danos ambientais e sociais decorrentes do acidente da barragem de Fundão, protagonizada pela mineradora Samarco, são da ordem de US\$ 521 milhões por ano. A perda estimada anual é em torno de seis vezes maior que todas as sete multas impostas pelo governo brasileiro. Outras multas ainda estão sendo discutidas, e incluem diferentes compensações econômicas, ambientais e sociais. Com base no exposto, é possível estabelecer a primeira hipótese deste estudo:

Hipótese 1: O acidente ambiental da mineradora Samarco impactou negativamente o retorno das ações de empresas mineradoras nos mercados brasileiro e australiano.

\subsection{Efeito dos acidentes ambientais sobre o risco sistemático do mercado de capitais}

O risco é um importante elemento na gestão estratégica e um fator determinante na performance das empresas. Kothari, Li e Short (2009) argumentam que, quando a análise do conteúdo dos relatórios indica evidenciações desfavoráveis, o risco da companhia (e.g., medido pelo custo de capital, volatilidade do retorno das ações e coeficiente beta) cresce significativamente.

Zreik e Louhichi (2017) explicam que existem três concepções de risco: total, sistemático e não sistemático. O risco total é a soma dos riscos sistemáticos e não sistemáticos. O risco sistemático, que reflete o retorno da resposta da companhia aos movimentos do mercado, é representado pelo coeficiente beta e afeta o mercado de modo mais amplo, com recessões econômicas, mudanças na taxa de juros ou aumento na inflação (Brigham \& Houston, 1999). Klassen e McLaughlin (1996) e Magness (2000) observaram mudanças no coeficiente beta usando a técnica de "estudos de eventos". Observou-se um declínio do coeficiente beta quando novas informações chegam ao mercado.

De acordo com Magness (2008), o declínio no coeficiente beta está relacionado parcialmente a um desajuste entre as ações da empresa e o mercado. Os investidores interpretam novas informações oriundas de um evento significativo e descontam no preço da ação. Além disso, a comunicação das empresas de alto risco sobre seu próprio risco está positivamente associada com o risco total e sistemático. Em contrapartida, para empresas de baixo risco, a comunicação diminui os três tipos de risco.

O risco não sistemático reflete a volatilidade do retorno das ações da empresa e não pode ser explicado pelos movimentos do mercado. Ele reflete a variabilidade do preço das ações de uma empresa e está associado com eventos ligados diretamente a ela. Um menor risco não sistemático está associado à questão ambiental no que diz respeito ao atendimento das expectativas dos stakeholders. Portanto, deve-se reduzir as externalidades e aumentar a evidenciação das práticas ambientais (Suchman, 1995).

Bansal e Clelland (2009) explicam que o risco não sistemático, que é afetado pelo comportamento dos investidores, tem uma relação com a legitimidade ambiental da empresa, que pode ser explicada sob a ótica da teoria institucional. Os autores defendem que a legitimidade ambiental de uma empresa é vista de forma diferente por todos os seus stakeholders, cada um considerando sua especificidade e ligação com a empresa. Como exemplo, explicam que a contaminação de uma fonte de água gera na comunidade vizinha uma preocupação relacionada à saúde, enquanto a preocupação de um acionista é uma multa que possa ser aplicada à empresa.

O risco não sistemático está associado a uma única empresa ou setor, e pode ser amenizado por meio de diversificação de portfólio, isto é, investimento em empresas com atividades diferentes. No entanto, Bonami e Lejeune (2009) mostram que, na prática, atingir 
essa diversificação é uma tarefa difícil devido a fatores como: número limitado de setores da economia, pouca rentabilidade advinda de pequenas participações em empresas e dificuldades na aquisição de ações.

Nesse sentido, Aaker e Jacobson (1987) apontam que, devido a essa dificuldade, investidores em geral preferem ações com baixo risco não sistemático, reduzindo a necessidade de diversificação. Nesse contexto, informações ambientais ganham uma grande relevância para os investidores, uma vez que os custos gerados na interação com o meio ambiente são, em geral, específicos para cada empresa, e há uma grande dificuldade de se antecipar eventos ambientais com impacto negativo (Bansal \& Clelland, 2009).

Por exemplo, a comunicação do risco sistemático, durante a crise financeira de 2008, aumentou o risco não sistemático e o risco total (Zreik \& Louhichi, 2017). A maioria dos investidores é avessa ao risco e interpreta a comunicação do risco de uma "empresa de alto risco" como um risco adicional. Por outro lado, empresas de baixo risco, quando divulgam seus riscos, são consideradas transparentes. Lokuwaduge e Heenetigala (2017) revelam que os relatórios podem criar reações negativas dos stakeholders. Estrategicamente, parece ser importante criar uma expectativa positiva nos stakeholders para gerenciá-los (ou manipulá-los) para obter sua aprovação ou distrair sua desaprovação. Com base no exposto, é possível estabelecer a segunda hipótese deste estudo:

Hipótese 2: O risco sistemático (coeficiente beta) diminuiu após o acidente ambiental da mineradora Samarco, para as empresas e os mercados brasileiro e australiano.

\section{METODOLOGIA}

\subsection{Seleção de empresas de mineração no mercado brasileiro e australiano}

Realizou-se uma amostragem das indústrias de mineração australianas e brasileiras baseadas em dois critérios: indústrias com a mesma classificação de atividade (exploração, desenvolvimento e produção de minerais) e que possuam ações listadas na BM\&FBovespa (e.g., empresas brasileiras) e na Australian Securities Exchange (ASX) (e.g., empresas australianas). No caso do mercado brasileiro, foram selecionadas todas as empresas do setor de mineração, o que totalizou quatro firmas. O mercado brasileiro é incipiente, tendo a Vale como líder, e as demais empresas operando como coadjuvantes.

Por outro lado, o mercado australiano é mais concorrido, e a disparidade na quantidade de empresas em relação ao Brasil exigiu a definição de um critério para seleção da amostra. Foi fixado um valor de mercado mínimo de $\mathrm{A} \$ 600 \mathrm{mi}$, com o objetivo de trabalhar com empresas de tamanhos similares, o que levou ao total de oito empresas australianas. Os efeitos da variação do preço das ações se tornam mais evidente neste grupo, contendo as maiores empresas australianas em valor de mercado.

\subsection{Estudo de eventos}

A resposta ao preço das ações é examinada usando a metodologia de "estudo de eventos". Campbell, Lo e Mackinlay (1996) definem estudo de eventos como o método pelo qual é possível medir o efeito de um evento econômico no valor de uma determinada empresa. Tal método avalia a racionalidade do mercado, considerando que o efeito de um evento será refletido imediatamente nos preços dos ativos.

Curran e Moran (2007) explicam que estudos de eventos são usados em pesquisas em contabilidade e finanças para avaliar o impacto no valor da firma em caso de mudanças na regulação ambiental ou em função de um processo legal que possa vir a causar danos à firma. De acordo com Binder (1998), o estudo de evento tem sido utilizado por duas principais razões: 1) testar a hipótese nula de que o mercado eficientemente incorpora informações; e 2) examinar o impacto de um determinado evento na riqueza dos acionistas de uma determinada empresa, tomando por base a "hipótese de mercado eficiente".

O estudo de eventos parte do pressuposto de que um acidente ambiental que afetou diretamente apenas uma única empresa desencadeará uma transferência de informações a 
toda a indústria. Esta transferência é sentida no mercado de capitais, com a oscilação do preço das ações (Clinch \& Sinclair, 1987). Indrabudiman (2017) explica que as reações no preço das ações podem ser medidas por meio dos retornos anormais, que consistem na variação dos preços dos ativos decorrida depois do evento (e.g., acidentes ambientais, fusões e aquisições).

Magness (2008) afirma que estudos de eventos são raros. Isto decorre, em parte, devido aos problemas estatísticos associados a pequenas amostras e à influência da própria dinâmica dos mercados. Além disso, baseia-se na hipótese de que os investidores descontarão "seu medo de uma reação legal" no preço da ação. Uma reação legal poderia significar uma suspensão do direito de operar. As empresas do setor industrial afetado poderiam enfrentar um aumento de demanda de informações, gerando um aumento dos custos de evidenciação.

\subsection{Cálculo dos retornos esperados}

Seguindo a metodologia proposta por Magness (2006), para o cálculo dos retornos das ações "sem o efeito do acidente" é fundamental escolher uma janela de tempo. Foi escolhida uma janela de "200 dias antes do acidente" e "200 dias após o acidente". O dia do acidente ambiental da mineradora Samarco (5/11/2015) foi fixado com o Dia 0. Os retornos diários da carteira de empresas são regredidos em relação ao retorno de mercado, com base no seguinte modelo econométrico:

$$
R_{i, t}=\beta_{0}+\beta_{1} \times R M_{p, t}+\varepsilon_{i, t}
$$

Onde $\mathrm{R}_{\mathrm{i}, \mathrm{t}}$ é o retorno da empresa i e t é a variação diária do preço da ação da empresa no tempo $t$, expressa em porcentagem; $\beta_{0}$ é o intercepto; $\beta_{1}$ representa o risco sistemático; RM é o retorno do mercado, t é a variação diária no mercado e $\varepsilon_{i, t}$ é o termo referente ao erro do modelo. Para o mercado australiano foi utilizado o retorno do S\&P/ASX 300, e para o mercado brasileiro foi adotado o retorno do Ibovespa. O objetivo desta análise é calcular os valores dos retornos sem o efeito do acidente.

Para avaliar o "efeito do acidente", a janela do cálculo dos retornos anormais foi reduzida para 100 dias. Partindo do "Dia 1", que é o primeiro dia comercial após o acidente ambiental da mineradora Samarco, até o "Dia 100", separando estratos de 10 em 10 dias. Nesta janela de 100 dias são comparadas as diferenças de médias dos "retornos sem o efeito do acidente" e os "retornos reais", usando o teste t de igualdade das médias. Esta análise identifica se houve o efeito de contágio do acidente na mineração brasileira e australiana.

Os retornos do portfólio são usados em vez do retorno individual da empresa, porque o modelo baseado no portfólio tem maior poder explicativo (Blacconiere \& Patten, 1994, p. 367). No entanto, incluir no portfólio as empresas diretamente afetadas pelo acidente pode influenciar os resultados. Isto acontece porque repercussões financeiras diretas (e.g. custos ambientais, custos de reparação dos danos e litígio) podem impactar o preço das ações da empresa. Por esta razão, foi feita a análise do retorno do portfólio com e sem as empresas Vale e BHP, responsáveis pelo acidente ambiental da mineradora Samarco.

Para medir o valor das empresas após o acidente, foi acrescentada a variável Dummy $D$, que assume valor igual a 0 para os dias antes da data do acidente, e 1 no dia do acidente $\mathrm{e}$ adiante. Esta variável permite capturar a parte do retorno total que é atribuído a uma mudança do beta no momento do acidente, conforme modelo a seguir:

$$
R_{i, t}=\beta_{0}+\beta_{1} \times R M_{p, t}+\beta_{2} \times R M_{p, t} \times D_{t}+\varepsilon_{i, t}
$$

A janela de tempo usada para o cálculo da perda de valor de mercado devida ao acidente foi de "200 dias antes do acidente" e "200 dias após o acidente". Se for correto assumir que após o acidente os acionistas reavaliaram a importância do gerenciamento ambiental no trade-off de risco/retorno, o coeficiente $\beta_{2}$ será significativo e menor do que o coeficiente $\beta_{1}$. 


\section{RESULTADOS}

A Tabela 1 apresenta o comparativo entre os "retornos sem o efeito do acidente" e os "retornos reais" na janela de 100 dias após o acidente, para os portfolios brasileiro e australiano com e sem as empresas responsáveis pelo acidente. Valores estatisticamente significativos revelam um comportamento anormal oriundo do acidente ambiental da mineradora Samarco. Os valores de significância permitem avaliar comportamentos do tipo "sobe e desce" das ações. Pode-se observar que, para o portfólio brasileiro, incluindo a empresa Vale, existe uma diferença significativa dos retornos entre o modelo "sem o efeito do acidente" e o "comportamento real das ações" para os dias 11 a 20, e 51 a 60 . Este resultado indica um efeito nas ações do mercado de mineração oriundo da divulgação do acidente ambiental da mineradora Samarco. Somente para a empresa Vale houve uma diferença significativa nos primeiros 10 dias, e nos dias 51-60 e 61-70. Quando o portfólio de empresas brasileiras é analisado sem a Vale, o modelo econométrico perde confiabilidade. Isto decorre do fato de a Vale ser a única empresa de mineração com ações negociadas diariamente na BM\&FBovespa.

Tabela 1

Nível de significância do teste de Wilcoxon entre o esperado e o observado das ações imediatamente após o Dia 1 e até 100 dias do acidente

\begin{tabular}{l|ccc|ccc}
\hline \multirow{2}{*}{ Período (dias) } & \multicolumn{3}{|c|}{ Portfólio Brasil } & \multicolumn{3}{c}{ Portfólio Austrália } \\
\cline { 2 - 7 } & Com a Vale & Somente a Vale & Sem a Vale & Com BHP & Somente BHP & Sem BHP \\
\hline $1-10$ & 0,3049 & $0,0658^{*}$ & $0,0526^{*}$ & 0,1598 & 0,1525 & 0,2099 \\
$11-20$ & $0,0371^{* *}$ & 0,4744 & $0,0253^{* *}$ & 0,2727 & 0,7596 & 0,4723 \\
$21-30$ & 0,4260 & 0,2610 & 0,2716 & 0,3832 & 0,2610 & 0,4260 \\
$31-40$ & 0,9095 & 0,1840 & 0,9698 & 0,3432 & $0,0079^{* * *}$ & 0,3060 \\
$41-50$ & 0,1826 & 0,9183 & 0,1588 & 0,9698 & 0,1525 & 0,9698 \\
$51-60$ & $0,0800^{*}$ & $0,0048^{* * *}$ & $0,0058^{* *}$ & $0,0477^{* *}$ & 0,4039 & $0,0477^{* *}$ \\
$61-70$ & 0,1209 & $0,0829^{*}$ & $0,0638^{*}$ & 0,7335 & $0,0248^{* *}$ & 0,5202 \\
$71-80$ & 0,0735 & 0,1823 & $0,0435^{* *}$ & 0,9090 & 0,6080 & 0,9090 \\
$81-90$ & 0,3432 & 0,6094 & 0,2399 & 0,1846 & 0,7592 & 0,1201 \\
$91-100$ & 0,2342 & 0,3526 & $0,0420^{* *}$ & 0,6200 & 0,2201 & 0,7330 \\
\hline
\end{tabular}

Nota. ${ }^{*} p<0,10 ;{ }^{* *} p<0,05 ;{ }^{* \star *} p<0,01$

Fonte: Dados da pesquisa coletados em Australian Securities Exchange (ASX) e a BM\&FBovespa.

Para o mercado australiano, o portfólio com a BHP mostra que a diferença do retorno das ações sem o acidente e o valor das ações é significativa para os dias 51 a 60 . Isto indica um efeito tardio afetando as ações do mercado de mineração australiano, oriundo da divulgação do acidente da mineradora Samarco. Analisando somente a BHP, observa-se que as ações da empresa foram afetadas antes (e.g., entre os dias 31 a 40) e depois (e.g., entre os dias 61 a 70) do efeito observado no mercado australiano. Retirando a BHP do portfólio australiano, as diferenças apresentam significância no mesmo período, ou seja, entre os dias 51 a 60. Este resultado confirma a maior diversificação do mercado de ações australiano no setor de mineração. O efeito nas ações de uma única empresa não consegue afetar o mercado, porque existem outras com o mesmo volume de negociações. Portanto, em relação a hipótese 1, o acidente provocou impacto negativo entre os dias 11 e 20 para o mercado de mineração brasileiro. Para o mercado australiano, o primeiro efeito foi sentido entre os dias 51 e 60.

A Tabela 2 mostra se houve uma mudança no risco não sistemático (beta) após o acidente para o mercado brasileiro. Os retornos do portfólio são regredidos em dois momentos usando a variável dummy, D. São observadas a magnitude e a significância dos betas $\beta_{1}$ e $\beta_{2}$ para cada empresa nos dois mercados, bem como para os mercados incluindo ou excluindo a empresa Vale. 
Tabela 2

Comparação do impacto nas empresas do mercado brasileiro entre 200 dias antes e 200 dias após o acidente ambiental da mineradora Samarco

\begin{tabular}{l|llllll}
\hline Empresas no Brasil & $\beta_{1}$ & Sig & $\beta_{2}$ & Sig & Teste F & $\mathrm{R}^{2}$ \\
\hline Vale & 1,098 & 0,000 & 0,663 & 0,006 & 59,440 & 0,308 \\
Bradespar & 0,606 & 0,000 & 1,261 & 0,000 & 64,050 & 0,325 \\
CCX & 0,439 & 0,066 & 0,301 & 0,320 & 1,351 & 0,010 \\
MMX & 0,444 & 0,656 & 0,551 & 0,663 & 0,475 & 0,004 \\
\hline Portfólio s/ Vale & 0,203 & 0,561 & 0,337 & 0,447 & 1,788 & 0,013 \\
Portfólio c/ Vale & 0,427 & 0,118 & 0,419 & 0,227 & 6,403 & 0,046 \\
\hline
\end{tabular}

Fonte: Dados da pesquisa coletados no BM\&FBovespa.

O portfólio das empresas de mineração no Brasil, incluindo ou não a principal empresa deste mercado (Vale) não apresentou significância, o que indica que o acidente não impactou o mercado brasileiro de mineração. Pode-se observar que a magnitude do coeficiente beta antes $\left(\beta_{1}\right)$ e após o acidente $\left(\beta_{2}\right)$ caiu para a empresa Vale, como era de se esperar, reforçando os resultados encontrados na Tabela 1. Entretanto, para a empresa Bradespar houve um comportamento ascendente, e para as empresas CCX e MMX houve uma alteração pouco significativa.

A Tabela 3 mostra a comparação do impacto do acidente ambiental da mineradora Samarco no mercado australiano. O coeficiente $\beta_{1}$ é significativo, e a magnitude dos coeficientes betas caiu para todas as empresas. Contudo, somente para as empresas BHP, Rio Tinto e NCM os valores de $\beta_{2}$ permanecem significativos. Este resultado indica que os efeitos do acidente ambiental da mineradora Samarco reduziram o valor das ações dessas empresas. Analisando o portfólio australiano com e sem a BHP, pode-se observar que o coeficiente $\beta_{2}$ só é significativo no portfólio com a BHP, observando-se uma redução de 1,073 no valor de $\beta_{1}$ para 0,277 no valor de $\beta_{2}$. Este resultado confirma a diversidade do mercado de ações australiano do setor de mineração, que apresenta várias opções de investimento.

Tabela 3

Comparação do impacto nas empresas no mercado australiano entre 200 dias antes e 200 dias após o acidente ambiental da mineradora Samarco

\begin{tabular}{|c|c|c|c|c|c|c|}
\hline Empresas na Austrália & $\beta_{1}$ & Sig & $\beta_{2}$ & Sig & Teste F & $\mathrm{R}^{2}$ \\
\hline $\mathrm{BHP}$ & 1,225 & 0,000 & 0,731 & 0,000 & 124,000 & 0,482 \\
\hline AWC & 1,074 & 0,000 & 0,331 & 0,102 & 50,850 & 0,276 \\
\hline Rio Tinto & 1,059 & 0,000 & 0,489 & 0,001 & 108,800 & 0,449 \\
\hline FMG & 1,485 & 0,000 & 0,473 & 0,214 & 27,290 & 0,170 \\
\hline NCM & 0,622 & 0,000 & 0,523 & 0,036 & 5,771 & 0,042 \\
\hline BSL & 1,277 & 0,000 & 0,066 & 0,812 & 27,740 & 0,172 \\
\hline SGM & 0,670 & 0,029 & 0,493 & 0,292 & 5,125 & 0,038 \\
\hline ILU & 1,177 & 0,000 & 0,287 & 0,190 & 48,920 & 0,268 \\
\hline Portfólio s/ BHP & 1,052 & 0,000 & 0,212 & 0,175 & 73,870 & 0,394 \\
\hline Portfólio c/ BHP & 1,073 & 0,000 & 0,277 & 0,067 & 86,720 & 0,390 \\
\hline
\end{tabular}

Fonte: Dados da pesquisa coletados em Australian Securities Exchange (ASX).

Observa-se a confirmação da hipótese 2 para as empresas e o mercado australiano. Houve declínio no risco sistemático beta para as empresas BHP, Rio Tinto e NCM, e para o portfólio com a BHP. No caso do Brasil, a hipótese 2 foi confirmada para a Vale e rejeitada para o mercado brasileiro como um todo.

\section{DISCUSSÃO}

O estudo adotou a metodologia de "estudo de eventos" para avaliar as respostas do mercado de capitais ao acidente ambiental da mineradora Samarco. Foram avaliadas as variações no retorno das ações e o risco sistemático (beta) das empresas controladoras BHP e Vale, e dos mercados em que elas operam. Os resultados permitem avaliar a dinâmica do ambiente institucional e os efeitos do acidente ambiental da mineradora Samarco no 
comportamento dos shareholders e investidores nos setores de mineração no Brasil e na Austrália.

Utilizando o modelo que apresenta os parâmetros de mercado para o retorno das ações das empresas de mineração brasileira e australiana, observou-se que, nos primeiros dias após o acidente, as ações da Vale sofrem perdas, e logo em seguida o mercado brasileiro como um todo é afetado. A divulgação das causas, dos efeitos e dos responsáveis gerou uma alteração nas ações da Vale e do mercado brasileiro. Mas depois de 60 dias do acidente o efeito do "sobe e desce" das ações não é mais sentido.

Para o mercado australiano (sem e com BHP), o efeito é significativo somente a partir dos dias 51 a 60 . As ações da BHP foram afetadas posteriormente à controladora brasileira. As empresas Vale e BHP não estavam associadas com a operação da mineradora Samarco. Corroborando este resultado, os estudos de Laplante e Lanoie (1994), no Canadá, afirmam que os preços das ações não respondem imediatamente quando uma empresa é citada por uma infração ambiental ou quando uma ação regulatória é incitada. O efeito significativo ocorre somente quando uma ação judicial resulta em multa.

A análise do risco sistemático (beta) demonstra que o mercado e as empresas no Brasil, com exceção da Vale, não apresentaram resultados significantes. Klassen e McLaughlin (1996) estudaram o coeficiente beta diante de vários eventos. Magness (2008) concluiu que o declínio no coeficiente beta está relacionado a um desajuste no preço das ações enquanto os investidores estão assimilando o evento, suas intercorrências e os efeitos gerados.

Os resultados para empresas do mercado australiano mostraram um declínio no risco sistemático beta somente para as empresas BHP, Rio Tinto e NCM. O mercado australiano parece ter sentido mais os efeitos do acidente ambiental da mineradora Samarco devido a suas características de competitividade e volatilidade. Estes resultados são consistentes com os achados de Lokuwaduge e Heenetigala (2017), no qual empresas de mineração australiana percebem pressões dos stakeholders para relatar informações sobre meio ambiente, segurança operacional e governança corporativa. Em consonância com a teoria da legitimidade e a teoria dos stakeholders, as empresas do setor de metais e mineração na Austrália divulgam estas informações de forma a reduzir o risco regulatório e salvaguardar sua legitimidade.

O mercado brasileiro de mineração apresenta uma baixa competitividade e limitadas opções de investimento. Segundo a consultoria internacional SNL, em 2014, de US\$11,4 bilhões investidos pela iniciativa privada em projetos de pesquisa mineral de não ferrosos, apenas 3\% foram carreados para o Brasil. Ao passo que outros países receberam um maior potencial de investimentos, tais como Chile (7\%), Peru (5\%), México (7\%), EUA (7\%), Austrália (12\%) e Canadá (14\%) (TRENDS, 2015). No Brasil, ainda se observa a presença da empresa Vale, operando em quase todo o mercado, e a empresa Bradespar, como a segunda maior empresa do mercado de mineração, que também investe na Vale. Outras duas empresas (CCX e MMX) se comportam de forma incipiente no mercado de mineração.

O estudo dos eventos ao longo de uma "janela de 200 dias" permite avaliar a influência do ambiente institucional sobre o comportamento dos tomadores de decisão da mineradora Samarco e suas controladoras. Avaliando as pressões regulatórias, Fonseca e Fonseca (2016) alertam que o arcabouço ambiental legal-normativo do Brasil, tem se mostrado frágil e em perigo de atuar sumariamente em defesa dos interesses corporativos. Um dos pilares da legislação ambiental, que reside no princípio da precaução, em que o Estado deve adotar medidas para evitar danos ambientais significativos, parece estar sendo negligenciado. A disfuncional divisão de poderes entre as empresas envolvidas e seus stakeholders se torna evidente na falta de controle das ações do Ministério Público e do Poder Judiciário.

Fracas pressões normativas e miméticas confirmam o atraso na resposta do mercado de ações. Este resultado sinaliza que fracos modelos de responsabilidade social corporativa são aceitos no setor de mineração. Os resultados desta pesquisa parecem indicar que a atuação da mineradora Samarco estava limitada ao escopo de sua "jurisdição gerencial", que traduz os vazios no ambiente institucional. Observa-se também a ausência dos atributos dos stakeholders, definidos por Mitchell et al. (1997). Investimentos éticos/sociais e/ou ambientais somente teriam sido realizados se o mercado de ações mandasse sinais claros às empresas do setor de mineração. As decisões da mineradora Samarco parecem ter sido orientadas por 
um fraco código de governança corporativa, que exclui demandas dos stakeholders e foca na maximização do retorno aos shareholders e investidores.

Uma questão relevante para explicar este "atraso" na resposta do setor de mineração envolve uma avaliação de como a mídia tratou o desastre ambiental causado pela mineradora Samarco. Fontes e Lopes (2017) destacam que a mídia, em um primeiro momento, não abordou de maneira aprofundada as questões relacionadas com os impactos ambientais (e.g., toxicidade da lama, abastecimento de água e impactos sobre a diversidade biológica da região afetada). Este comportamento pode ser explicado pela falta de conhecimento, ou até mesmo ser intencional, em função de interesses das empresas controladoras da mineradora Samarco.

No entanto, o enfoque sensacionalista se mostrou presente, e sempre minimizando as responsabilidades das empresas Samarco, Vale e BHP e suas possíveis consequências. Desse modo, a mídia transmite a informação à sociedade mas sem apresentar as relações existentes entre a atividade mineradora, os danos ambientais e as questões sociais. Garcia et al. (2017) alertam que o afrouxamento de certas leis brasileiras e a concessão de novas áreas de exploração estão sendo realizadas sem um claro diálogo com a sociedade.

\section{CONCLUSÃO}

O estudo de eventos revelou que houve um impacto negativo imediato logo após o acidente apenas para as ações da Vale. O mercado brasileiro sentiu este efeito depois de 10 dias, e o mercado australiano depois de 50. Somente com a divulgação dos detalhes do acidente é que pôde ser visto um efeito tardio e temporário nas ações das empresas controladoras e em seus mercados. No entanto, os impactos econômicos, ambientais e sociais foram rapidamente absorvidos pelo mercado de capitais, no Brasil e na Austrália, e não influenciaram o valor das ações de empresas de mineração a longo prazo.

Em relação à hipótese do risco sistemático (beta), os achados da pesquisa revelam que houve um declínio do coeficiente para as empresas Vale, BHP, Rio Tinto e NCM. Os achados da pesquisa confirmam o argumento de que em mercados mais competitivos há uma tendência pela busca de legitimidade. Conforme argumentam Pellegrino e Lodhia (2012), há um contrato social entre as organizações e a sociedade baseado nas expectativas implícitas e explicitas que a sociedade possui sobre a condução das operações das empresas.

Deve-se reconhecer como fator limitante desta pesquisa o restrito número de empresas e a situação bem particular do mercado brasileiro de mineração, com apenas uma empresa líder (Vale), uma investidora (Bradespar) e duas empresas (MMX e CCX) com atuação incipiente no mercado de mineração. Outra limitação envolve a quantidade de variáveis explicativas no modelo econométrico para o cálculo do retorno das ações. Apesar das limitações, o trabalho alerta para a necessidade de ampliar as pressões dos investidores sobre o setor de mineração. Esta pressão deve garantir que mineradoras atuem de forma responsável, e que os traumas deste desastre não se prologuem nem se repitam.

\section{REFERÊNCIAS}

Aaker, D. A., \& Jacobson, R. (1987). The role of risk in explaining differences in profitability. Academy of Management Journal, 30(2), 277-296.

Abreu, M. C. S, Cunha, L. T., \& Barlow, C. Y. (2015). Institutional dynamics and organizations affecting the adoption of sustainable development in the United Kingdom and Brazil. Business Ethics: a European Review, 24(1), 73- 90.

Abreu, M. C. S., Varvakis, G., \& Figueiredo, H. S., Júnior (2004). As pressões ambientais da estrutura da indústria. RAE-eletrônica, 3(2), 1-31.

Alpaslan, C, Green, S. E., \& Mitroff, I. I. (2009). Corporate governance in the context of crisis: towards a stakeholder theory of crisis management. Journal of Contingencies and Crisis Management, 17(1), 38-49. 
Bansal, P., \& Clelland, I. (2004). Takling trash: legitimacy impression management and unsystematic risk in the context of the natural environment. Academy of Management Journal, 41(1), 93-103.

Baskerville-Morley, R. (2004). Dangerous, dominant, dependente or definifive: stakeholder identification when profession faces major transgressions. Accounting and the Public Interest, 4, 24-42.

Binder, J. J. (1998). The event study methodology since 1969. Review of Quantitative Finance and Accounting, 11(2), 111-137.

Blacconiere, W., \& Patten, D. (1994). Environmental disclosures, regulatory costs and changes in firm value. Journal of Accounting and Economics, 18, 357-377.

Blanco, E., Maquieira, J., \& Lozano, J. (2009). The economic impacts of voluntary environmental performance of firms: a critical review. Journal of Economic Surveys, 23(3), 462-502.

Bonami, P., \& Lejeune, M. A. (2009). An exact solution aroach for portfolio optimization problems under stochastic and integer constraints. Operations research, 57(3), 650-670.

Brigham, E. F., \& Houston, J. F. (1999). Fundamentos da moderna administração financeira. Campus.

Campbell, J. L. (2007). Why would corporations behave in socially responsible ways? An institutional theory of corporate social responsibility. Academy of management Review, 32(3), 946-967.

Campbell, J. Y., Lo, A. W., \& Mackinlay, C. A. (1996), The econometrics of financial markets. Princeton University Press.

Clarkson, M. E. (1995). A stakeholder framework for analyzing and evaluating corporate social performance. Academy of management review, 20(1), 92-117.

Clinch, G. J., \& Sinclair, N. A. (1987). Intra-industry information releases: A recursive systems approach. Journal of Accounting and Economics, 9(1), 89-106.

Curran, M. M., \& Moran, D. (2007). Impact of the FTSE4Good Index on firm price: An event study. Journal of Environmental Management, 82(4), 529-537.

Deegan, C. (2002) Introduction: the legitimizing effect of social and environmental disclosures a theoretical foundation. Accounting, Auditing \& Accountaility Journal, 15(3), 282-311.

Delmas, M., \& Toffel, M. W. (2004). Stakeholders and environmental management practices: an institutional framework. Business strategy and the Environment, 13(4), 209-222.

DiMaggio, P., \& Powell, W. W. (1983). The iron cage revisited: Collective rationality and institutional isomorphism in organizational fields. American sociological review, 48(2), 147-160.

Elliott, M. R., Wang, Y., Lowe, R. A., \& Kleindorfer, P. R. (2004). Environmental justice: frequency and severity of US chemical industry accidents and the socioeconomic status of surrounding communities. Journal of Epidemiology \& Community Health, 58(1), 24-30. 
Espíndola, H. S., Campos, R. B. F, Lamounir, K. C. C., \& Silva, R. S. (2016) Desastre da Samarco no Brasil: desafios para a conservação da biodiversidade. Journal of Social, Technological and Environmental Science, 5(3), 72-100.

Fonseca, P. G. \& Fonseca, G. I. (2016). Brazil's greatest environmental catastrophe Samarco's Fundão Tailings Dam. Environmental Policy and Law, 46(5), 334-337.

Fontes, R. N. \& Lopes, I. S. (2017). As representações da mídia sobre o rompimento da barragem do Fundão em Mariana, Minas Gerais. Espacios, 38(34), 32-46.

Garcia, L. C., Ribeiro, D. B., Oliveira Roque, F., Ochoa-Quintero, J. M., \& Laurance, W. F. (2017). Brazil's worst mining disaster: corporations must be compelled to pay the actual environmental costs. Ecological alications, 27(1), 5-9.

Goebel, J., Krekel, C., Tiefenbach, T., \& Ziebarth, N. R. (2015). How natural disasters can affect environmental concerns, risk aversion, and even politics: evidence from Fukushima and three European countries. Journal of Population Economics, 28(4), 1137-1180.

Heflin, F., Moon Jr, J. R., \& Wallace, D. (2015). A re-examination of the cost of capital benefits from higher-quality disclosures. Journal of Financial Reporting, 1(1), 65-95.

Henriques, I., \& Sadorsky, P. (1996). The determinants of an environmentally responsive firm: an empirical aroach. Journal of environmental economics and management, 30(3), 381395.

Hoffman, A. J. (2001). From heresy to dogma: An institutional history of corporate environmentalism. Stanford University Press.

Indrabudiman, A. (2017). Does social and environmental disclosure affect stock price? Evidence from Indonesian. International Journal of Economic Researach, 14(4), 419-428.

Jamali, D., \& Mirshak, R. (2007). Corporate social responsibility (CSR): Theory and practice in a developing country context. Journal of business ethics, 72(3), 243-262.

Jamali, D., \& Neville, B. (2011). Convergence versus divergence of CSR in developing countries: An embedded multi-layered institutional lens. Journal of Business Ethics, 102(4), 599-621.

Jenkins, H., \& Yakovleva, N. (2006). Corporate social responsibility in the mining industry: exploring trends in social and environmental disclosure. Journal of Cleaner Production, 14(3), 271-284.

Jennings, P. D., \& Zandbergen, P. A. (1995). Ecologically sustainable organizations: An institutional aroach. Academy of management review, 20(4), 1015-1052.

Karpoff, J. M., Lott, J. R., Jr., \& Wehrly, E. W. (2005). The reputational penalties for environmental violations: empirical evidence. Journal of Law and Economics, 48(2), 653675.

Klassen, R. \& McLaughlin, C. (1996). The impact of environmental management on firm performance. Management Sciente, 42, 1199-1214.

Klerk, M., \& Villiers, C. de (2012). The value relevance of corporate responsibility reporting: South African evidence. Meditari Accountancy Research, 20(1), 21-38. 
Kothari, S. P., Li, X., \& Short, J. E. (2009). The effect of disclosures by management, analysts, and business press on cost of capital, return volatility, and analyst forecasts: A study using content analysis. The Accounting Review, 84(5), 1639-1670.

Kumar, R., Lamb, W. B., \& Wokutch, R. E. (2002). The end of South African sanctions, institutional ownership, and the stock price performance of boycotted firms: Evidence on the impact of social/ethical investing. Business \& Society, 41(2), 133-165.

Laplante, B., \& Lanoie, P. (1994). The market response to environmental incidents in Canada: a theoretical and empirical analysis. Southern Economic Journal, 657-672.

Lin, P. T., Li, B., \& Bu, D. (2015). The relationship between corporate governance and community engagement: Evidence from the Australian mining companies. Resources Policy, 43, 28-39.

Lokuwaduge, C. S. D. S., \& Heenetigala, K. (2017). Integrating environmental, social and governance (ESG) disclosure for a sustainable development: an Australian study. Business Strategy and the Environment, 26(4), 438-450.

Magness, V. (2000). The impact of environmental accidents on the behavior of TSE traded companies. Unpublished Doctoral Dissertation (University of Manitoba).

Magness, V. (2008). Who are the stakeholders now? An empirical examination of the Michell, Agle and Wood theory of stakeholder salience. Journal of Business Ethics, 83(2), 1771992.

Meyer, J. W., \& Scott, W. R. (1992). Organizational environments: Ritual and rationality. Sage Publications, Inc.

Milstein, M. B., Hart, S. L., \& York, A. S. (2002). Coercion breeds variation: the differential impact of isomorphic pressures on environmental strategies (pp. 151-172). Stanford University Press: Stanford.

Mitchell, R. K., Agle, B. R., \& Wood, D. J. (1997). Toward a theory of stakeholder identification and salience: defining the principle of who and what really counts. Academy of Management Review, 22(4), 853-886.

Morgan, G., Gomes, M. V. P., \& Perez-Aleman, P. (2016). Transnational governance regimes in the global south: multinationals, states and NGOs as political actors. Revista de Administração de Empresas, 56(4), 374-379.

Pargai, S., \& Wheeler, D. (1995). Informal regulation of industrial pollution in developing countries: evidence from Indonesia. Workd Bank, Policy and Research Department Working Paper, 1416.

Patten, D. M. (1992). Intra-industry environmental disclosures in response to the Alaskan oil spill: a note on legitimacy theory. Accounting, organizations and Society, 17(5), 471-475.

Patten, D. M., \& Trompeter, G. (2003). Corporate responses to political costs: an examination of the relation between environmental disclosure and earnings management. Journal of Accounting and Public Policy, 22(1), 83-94.

Pellegrino, C., \& Lodhia, S. (2012). Climate change accounting and the Austratian mining industry: exploring the links between corporate disclosure and the generation of legitimacy. Journal of Cleaner Production, 36, 68-82. 
Siltaoja, M. E., \& Onkila, T. 2013. 'Business in society or business and society: the construction of business-society relations in responsibility reports from a critical discursive perspective'. Business Ethics: A European Review, 22(4), 357-373.

Suchman, M. C. (1995). Managing legitimacy: Strategic and institutional aroaches. Academy of management review, 20(3), 571-610.

Visschers, V. H., \& Siegrist, M. (2012). Fair play in energy policy decisions: Procedural fairness, outcome fairness and acceptance of the decision to rebuild nuclear power plants. Energy Policy, 46, 292-300.

Yamaguchi, K. (2008). Reexamination of stock price reaction to environmental performance: a GRACH alication. Ecologial Economics, 68(1), 345-352.

Zreik, O., \& Louhichi, W. (2017). Risk Disclosure and Company Unsystematic, Systematic, and Total Risks. Economics Bulletin, 37(1), 448-467. 